\title{
Category-Theoretic Interpretative Framework of the Complementarity Principle in Quantum Mechanics
}

\author{
Elias Zafiris and Vassilios Karakostas
}

\begin{abstract}
This study aims to provide an analysis of the complementarity principle in quantum theory through the establishment of partial structural congruence relations between the quantum and Boolean kinds of event structure. Specifically, on the basis of the existence of a categorical adjunction between the category of quantum event algebras and the category of presheaves of variable Boolean event algebras, we establish a twofold complementarity scheme consisting of a generalized/global and a restricted/local conceptual dimension, where the latter conception is subordinate to and constrained by the former. In this respect, complementarity is not only understood as a relation between mutually exclusive experimental arrangements or contexts of co-measurable observables, as envisaged by the original conception, but it is primarily comprehended as a reciprocal relation concerning information transfer between two hierarchically different structural kinds of event structure that can be brought into partial congruence by means of the established adjunction. It is further argued that the proposed category-theoretic framework of complementarity naturally advances a contextual realist conceptual stance towards our deeper understanding of the microphysical nature of reality.
\end{abstract}

1. On the Structure of the Complementarity Concept

2. Universal Solution to the Functorial Representation of a Quantum Event Algebra via Boolean Frames

\subsection{Conceptual framework}

2.2 Functorial representation of Quantum event algebras via Boolean frames

3. Twofold Complementarity Scheme via the Boolean Frames - Quantum Adjunction

3.1 Towards a twofold conception of complementarity

3.2 Global conceptual dimension of complementarity

3.3 Local conceptual dimension of complementarity

4. Conceptual Aspects of the Twofold Complementarity Scheme

5. Appendix

\footnotetext{
${ }^{0}$ To contact the authors, please write to: Elias Zafiris, Parmenides Foundation, Center for the Conceptual Foundations of Science, Kirchplatz 1, Pullach, 82049 Munich, Germany; e-mail: ezafiris@math.uoa.gr, or to Vassilios Karakostas, Department of Philosophy and History of Science, Faculty of Sciences, University of Athens, Athens 157 71, Greece; e-mail: karakost@phs.uoa.gr.
} 


\section{On the Structure of the Complementarity Concept}

The concept of complementarity was introduced in quantum mechanics by Niels Bohr in his lecture at the International Congress of Physics, held in the Italian city of Como, in 1927, although the essential ideas reach back to 1925 . He proposed the complementarity doctrine as the new framework for the ordering of physical experience in the microscopic domain. Apart from being conceived as an inseparable aspect of quantum theory, Bohr himself envisaged his views on complementarity as forming also part of a wider regulative epistemological principle which is required whenever a sharp distinction between the (observing) subject and the (observed) object cannot be made. The closing sentence of his Como lecture is telling: "I hope, however, that the idea of complementarity is suited to characterize the situation, which bears a deep-going analogy to the general difficulty in the formation of human ideas, inherent in the distinction between subject and object" $[5$, p. 590].

In his numerous articles on the topic, Bohr strove to develop the concept of complementarity into a definite viewpoint, nonetheless, he never provided an exact definition or a sharp formulation [26, Sect. 3]. Instead, he expressed the concept of complementarity qualitatively in various terms on different occasions, according to the physical situation under consideration. In the early phase of the interpretation of quantum physics, Bohr used complementarity to characterize a binary relation between, for example, physical pictures such as the particle picture and the wave picture, or physical variables such as position and momentum, or physical descriptions such as the space-time description and the dynamical description based on the consideration of conservation laws. As a view about the nature of physical pictures, complementarity declares that each atomic system has a wave aspect and a particle aspect both of which being equally fundamental from a classical viewpoint but yet mutually incompatible within a single quantum phenomenon. As a view about the assignment of physical variables, it designates that conjugate quantities, like position and momentum, cannot be ascribed exact simultaneous values; the corresponding quantitative limits are to be specified by Heisenberg's indeterminacy relations. As a view about physical descriptions, it asserts that there are mutually exclusive conditions for the unambiguous use of the kinematical concepts involving detailed space-time coordination and dynamical concepts involving conservation laws of momentum and energy (the latter sometimes being referred to as a "requirement of causality"). The incompatibility between spatio-temporal $(t, \mathbf{r})$ and dynamical concepts $(E, \mathbf{p})$ is traced back to the unavoidable presence of Planck's quantum of action in the atomic domain, imposing an in principle uncontrollable transfer of momentum and energy to fixed scales and synchronized clocks needed to define the reference frame for specifying exact trajectories.

In his later writings, demarcated essentially by the time of Bohr's [6] reply to the EPR incompleteness argument, his viewpoint of complementarity gradually converges towards a preferred expression of complementarity as a relationship between phenomena demanding mutually exclusive experimental arrangements. No doubt, Bohr's concept of a quantum phenomenon forms the logical basis for his expounded notion of complementarity. According to Bohr, the fundamental difference with respect to the analysis of phenomena in classical and in quantum physics is that in the latter the 'measured system', the 'measuring apparatus' and their 'mutual interaction' constitute an indivisible whole in such a manner that analysis into disjoint elements is not admissible without destroying or altering the original phenomenon. "The essential wholeness of a proper quantum phenomenon", writes 
Bohr, "finds indeed logical expression in the circumstance that any attempt at its welldefined subdivision would require a change in the experimental arrangement incompatible with the appearance of the phenomenon itself" [7, p. 291]. In this respect, he strongly advocated that the use of the word "phenomenon" must exclusively refer to "observations obtained under specified circumstances, including an account of the whole experiment" [9, p. 317]. Thus, for Bohr, a quantum phenomenon is not fully specified until the whole experimental procedure is determined.

The general epistemological thesis advanced by Bohr towards this direction, especially as regards the ordering and subsequent synthesis of phenomena, consists in conditionalizing the meaning of theoretical concepts to the context of a possible experiment. It is only by knowing the experimental conditions that the concepts used for the description of experimental results can become relevant in the context of a physical inquiry.

In this connection, Bohr clearly realized that an unambiguous interpretation of the quantum mechanical formalism can be achieved only by an equally unambiguous description of the phenomena, with the experimental arrangement specified in classical terms, at least to the degree that it functions as a measuring instrument:

In the system to which the quantum mechanical formalism is applied, ... some ultimate measuring instruments, like the scales and clocks which determine the frame of space-time coordination on which, in the last resort, even the definitions of momentum and energy quantities rest must always be described entirely on classical lines, and consequently kept outside the system subject to quantum mechanical treatment.

... It is just in this sense that phenomena defined by different concepts, corresponding to mutually exclusive experimental arrangements, can unambiguously be regarded as complementary aspects of the whole obtainable evidence concerning the objects under investigation [8, pp. 316-317].

With this terminology, the complementary aspects between conjugate variables, waveparticle pictures, or physical descriptions, as previously specified, can be derived from a primary notion of complementarity, by referring to phenomena appearing under mutually exclusive experimental arrangements, constituting also Bohr's ultimate formulation on the issue. In Bohr's own words:

The impossibility of combining phenomena observed under different experimental arrangements into a single classical picture implies that such apparently contradictory phenomena must be regarded as complementary in the sense that, taken together, they exhaust all well-defined knowledge about the atomic objects [10, p. 25].

Bohr's preceding expression of "apparently contradictory phenomena", elsewhere being characterized as "apparently incompatible" [7, p. 291], when referred to complementary aspects of the same object, can only mean incompatible on classical considerations alone. Any two phenomena, then, can be called complementary if, by referring to the same object, they are mutually exclusive in the sense that the two corresponding experimental arrangements employed for the manifestation of the phenomena and the information which they yield are incompatible, while at the same time being jointly necessary for an exhaustive as possible description of the object concerned as allowed by quantum theory. 
Despite divergent views in the philosophical outlook of Bohr's approach to quantum mechanics, as offered by various Bohr scholars [4, 22, 27, 40], there is a consensus in recent research literature that the combination of the two aforementioned features concerning "mutual exclusiveness" and "joint completion" captures the gist of quantum complementarity $[14,18,36,41]$. As an illustration, let us consider concisely the most characteristic example of complementary phenomena involving the measurement of the position and momentum observables. According to the original conception of complementarity, position and momentum cannot both be sharply defined in one and the same measurement context. For, the context needed for a measurement of the position observable is not compatible with that needed for a measurement of the momentum observable. This suggests that they should be measured independently, using mutually exclusive experimental arrangements, thus rendering the notions of position and momentum complementary in the sense that they are not simultaneously applicable as precisely defined quantities. Yet, this holds true for any conjugate pair of observables $A$ and $B$ satisfying the canonical commutation relation $[A, B]=i \hbar I$. The existence of complementary physical quantities and relations is ubiquitous, reflecting an essential feature of the quantum mechanical formalism. In this respect, the complementarity principle may be viewed as a distinct consequence of it, highlighting certain remarkable implications of the theory, suitably specified. If, therefore, the complementarity concept is projected into the Hilbert space formulation of quantum mechanics, we arrive at an explicit characterization of the functioning of complementarity in quantum theory that may attract general acceptance. On this basis, the structure of the hard core of complementarity may be decomposed as follows:

(i) A quantum mechanical observable is well-defined within the experimental arrangement or measurement context serving to measure that observable.

(ii) Upon specifying an experimental arrangement and, therefore, selecting an observable to be measured, atomic propositions referring to the properties of a quantum mechanical system acquire, within the associated measurement context, determinate truth values [33], thus, forming a Boolean subalgebra in the non-Boolean logical structure of the system.

(iii) Simultaneous assignment of exact or sharp values to pairs of complementary observables, be it by preparation or measurement procedures, is impossible. Nonetheless, simultaneous assignment of approximate or unsharp values to such observables is feasible within the limits allowed by the uncertainty or indeterminacy relations. ${ }^{1}$

(iv) Complementary sharp observables correspond to different mutually exclusive experimental arrangements, defining different aspects of reality, which cannot be unified in a single classical Boolean picture.

(v) Each complementary aspect of reality, thus defined, is jointly necessary for a comprehensive account of the quantum phenomenon.

\footnotetext{
${ }^{1}$ The possibility of describing simultaneous (or joint) unsharp measurements of pairs of complementary observables requires the extension of the standard formalism of quantum mechanics by allowing observables to be represented by positive-operator-valued measures (POVMs). The consideration of a POVM characterizes a "coarse grained" or "smeared" version of a sharp observable providing, in effect, representations of observables describing imperfect or approximate joint measurements. As expected, the measuring inaccuracies imposed in such joint measurements ought to be in accordance with Heisenberg's indeterminacy relations. For a general introduction to the formalism of POVMs, the interested reader may consult [13].
} 
It is worth noting that in recent years the complementarity concept has been shown to underlie many aspects of quantum information technology, ranging from quantum superdense coding [17] to information complementarity [51] and from the security of quantum cryptography [23] to quantitative complementarity relations in interferometry [12] and delayed-choice experiments [38]. In this respect, our attempt to expand and accommodate the idea of complementarity within a formal description of quantum mechanics may have implications in all preceding areas.

In this work we aim to establish that the concept of complementarity, pertaining to any non-Boolean quantal physical description, is mathematically formalized, conceptually further clarified and physically extended, through the categorical notion of adjunction that has been recently proved to exist between the category of quantum event algebras and the category of presheaves on Boolean event algebras (Zafiris [45], see also Zafiris \& Karakostas [50] for a more detailed treatment including in addition the involved logical aspects). To this end, in Section 2, we provide a category-theoretic representation of a quantum algebra of events via the adjunction of suitably qualified functors of Boolean probing frames to it, capable of carrying all the information encoded in the former. In Section 3, we demonstrate that this Boolean frames-quantum adjunction embodies the semantics of complementarity and, furthermore, explicates its functioning through a twofold scheme consisting of a generalized/global and a restricted/local conceptual dimension. The crucial notion of adjunction, which does not have a set-theoretic counterpart, provides the mathematical means of relating relations and, thus, establishing local or partial structural congruences between the quantum and Boolean kinds of event structure. It is precisely this fact which allows an extended interpretation of the quantum complementarity principle by relating internally and bi-directionally the variable and local Boolean with the quantum global level of structure. In light of the proposed interpretative framework, complementarity is not only understood as a relation between mutually exclusive experimental arrangements or contexts of co-measurable observables, as envisaged by the original conception, but it is primarily comprehended as a reciprocal relation concerning information transfer between two hierarchically different structural kinds of event structure-the Boolean and quantum kinds of structure-that can be brought into partial congruence by means of the established adjunction. Finally, in Section 4, we explicate the global and local dimension of complementarity, clarify their interdependence, and develop certain further conceptual aspects of the twofold complementarity scheme. We also include an appendix where we briefly compare our category-theoretic representation scheme of quantum event algebras with other categorial approaches by putting emphasis on the complementarity issue.

\section{Universal Solution to the Functorial Representation of a Quantum Event Algebra via Boolean Frames}

\subsection{Conceptual framework}

The complementarity scheme developed in the sequel is motivated by the physically significant observation that whereas the totality of all experimental/empirical facts associated to a quantum mechanical system can only be represented in a globally non-Boolean logical structure, the acquisition of every single fact depends on a locally Boolean context. An intuitive understanding of this insight may be advanced by pointing out that although 
Kochen-Specker's [35] theorem in quantum logic excludes the possibility of a global twovalued truth-functional assignment on the non-Boolean structure of a quantum system, nonetheless, there always exists a local two-valued truth-functional assignment with respect to a complete Boolean algebra of projection operators on the Hilbert space of the system. In more detail, in virtue of the spectral resolution theorem for self-adjoint operators, each observable, represented by such an operator, is associated with the Boolean algebra of projection operators that spectrally resolve it, identified in terms of a local Boolean subalgebra of the global non-Boolean event algebra of a quantum system. Therefore, given a set of observables of a quantum system, there always exists a complete Boolean subalgebra of projection operators with respect to which a local two-valued truth-functional assignment is viable, if and only if the given observables commute or, equivalently, they are simultaneously measurable. It should be underlined that such a complete Boolean algebra of projection operators bears the status of a logical structural invariant characterizing a whole commutative algebra of observables that can be simultaneously spectrally resolved, and hence be value definite. Since in the lattice of quantum events there exist complementary or incompatible physical magnitudes non-commuting with any considered commutative algebra of observables, there exists a multiplicity of possible Boolean algebras of orthogonal projections furnishing an invariant of this kind only in the context of all commuting observables in question.

In this manner, the conceptual framework surrounding the notion of complementarity should pertain to the fact that in the quantum domain there does not exist a unique and universal logical structural invariant with respect to which all possible observables can be spectrally resolved simultaneously, but, in contradistinction, there is a multiplicity of such invariants attached only to commutative subalgebras of observables. Therefore, although a quantum event structure is globally non-Boolean, it can be qualified spectrally, and hence be accessed experimentally, only in terms of Boolean event structures operating as logical structural invariants of co-measurable families of physical magnitudes (cf. also Svozil [43, Sect. 12.9.11]). Consequently, a complete Boolean algebra of projection operators in its function as a spectral invariant of a commutative subalgebra of quantum observables furnishes the role of a Boolean logical frame with respect to which a quantum event can be qualified and lifted to the empirical level. Thus, the consideration of each local Boolean frame at any temporal moment serves as a natural pre-condition for establishing a local invariant logical structure for the event evaluation of all co-measurable observables forming this context. Due to the absence of a global, uniquely defined Boolean frame over a quantum event structure, it is necessary to consider all possible local ones together with their interrelations. From this perspective, the crucial problem is if there exists a universal way to specify a quantum event structure via the literal adjunction of local spectral invariants to it, objectified by variable local Boolean frames, under their intended physical interpretation as probing frames for the manifestation and concomitant classification (or contextualization) of quantum events.

We advance the view that the conceptual net of ideas enveloping the notion of complementarity pertains exactly to the possibility of existence of a universal solution to the preceding problem. The existence of a universal solution essentially renders the global orthomodular lattice structure of quantum events empirically inert without the adjunction of local spectral invariants to it. The role of these invariants is to induce partial or local structural congruences with Boolean event structures pertaining to all typical contexts of measurement. Intuitively, the multiplicity of applicable local Boolean frames allows the 
filtration and separation of several resolution sizes and types of quantum observable grain depending on the qualification of the corresponding spectral orthogonal projections. The objective of a universal solution is to derive the non-directly accessible quantum kind of event structure by means of all possible partial structural congruences with the directly accessible Boolean kind of event structure, forced by means of adjoining local spectral invariants as probing frames to the former. In this setting, the major role is subsumed by all possible structural relations allowed among the probing Boolean frames, the spectra of which may be disjoint or nested or overlapping and interlocking together nontrivially. The condition that distinct Boolean frames may have nontrivial intersections or, more generally, nontrivial pullback compatibility relations with respect to a global quantum event structure partly extends the semantics of complementarity beyond the standard standpoint concerning disjoint Boolean frames. As outlined however in Sect. 1, it is the proposed category-theoretic analysis of quantum complementarity, based on the existence of a categorical adjunction between the category of quantum event algebras and the category of presheaves of variable Boolean event algebras, which broadens the scope of the complementarity concept and is pertinent to the present approach. In turn, the universality of the existence of such a categorical adjunction poses the necessity to formulate the problem in functorial terms, i.e., non-dependent on the artificial choice of particular Boolean frames adjoined to a quantum event algebra.

\subsection{Functorial representation of quantum event algebras via Boolean frames}

Category theory provides a general theoretical framework for the study of structured systems in terms of their mutual relations and admissible transformations. Contrary to the atomistic approach of set theory which crucially depends on the concept of elements-points and the membership relationship of a variable $x$ in a set $X, x \in X$, in category theory the notion of morphism or arrow undertakes primary role. A morphism, for instance, $f: A \rightarrow B$ in a category $\mathcal{C}$ expresses one of the many possible ways in which the object $A$ relates to the object $B$ within the context of category $\mathcal{C}$. However counterintuitive it may initially appear, in category theory the nature of the objects is a derivative aspect of the patterns described by the morphisms or mappings that connect the objects. In fact, an object can be completely classified and uniquely derived up to an isomorphism by the network of all morphisms - the structure preserving relations - targeting this object within the same category. Most significantly, this does not exclude the inter-level relational determination of objects belonging to other categorical species of structure, under the condition that there exists a bi-directional functorial correlation between them, formulated in the language of adjunctions. It is precisely the latter development that gradually introduced into category theory a paradigm change in understanding structures of general types and paved the way for forming bridges between seemingly unrelated mathematical disciplines.

The basic categorical principles that we adopt in the subsequent analysis are summarized as follows:

(i) To each kind of mathematical structure used to represent a system, there corresponds a category whose objects have that structure and whose arrows or morphisms preserve it. 
(ii) To any natural construction on structures of one kind, yielding structures of another kind, there corresponds a functor from the category of the first specified kind to the category of the second. The implementation of this principle is associated with the fact that a categorical functorial construction is not merely a function from objects of one kind to objects of another kind, but must preserve the essential structural relationships among objects, that is, identity morphisms and composition of morphisms.

(iii) To each natural translation between two functors having identical domains and codomains, there corresponds a natural transformation. The specification "natural" in the notion of natural transformations refers to the comparison of two functorial processes, sharing the same source and target categories, in a way that captures the shared structure or generic common properties existing in different categorical contexts.

(iv) To any canonical bi-directional functorial correlation between two kinds of mathematical structures, there corresponds an adjunction realized by a pair of adjoint functors between the corresponding categories. ${ }^{2}$

In view of the aforementioned principles, the functorial representation of a quantum event algebra in terms of suitably qualified functors of Boolean probing frames requires distinct notions of Boolean and quantum categorical event structures, respectively.

A Boolean categorical event structure is a small category, denoted by $\mathcal{B}$, which is called the category of Boolean event algebras. The objects of $\mathcal{B}$ are complete Boolean algebras of events and the arrows are the corresponding Boolean algebraic homomorphisms.

A quantum categorical event structure is a locally small co-complete category, denoted by $\mathcal{L}$, which is called the category of quantum event algebras. The objects of $\mathcal{L}$ are quantum event algebras and the arrows are quantum algebraic homomorphisms.

A quantum event algebra $L$ in $\mathcal{L}$ is defined as an orthomodular $\sigma$-orthoposet [15], that is, as a partially ordered set of quantum events, endowed with a maximal element 1 , and with an operation of orthocomplementation $[-]^{*}: L \rightarrow L$, which satisfy, for all $l \in L$, the following conditions: [a] $l \leq 1,[\mathrm{~b}] l^{* *}=l$, [c] $l \vee l^{*}=1,[\mathrm{~d}] l \leq \hat{l} \Rightarrow i^{*} \leq l^{*}$, [e] $l \perp \dot{l} \Rightarrow l \vee i \in L,[\mathrm{f}]$ for $l, i \in L, l \leq l$ implies that $l$ and $\dot{l}$ are compatible, where $0:=1^{*}$, $l \perp i l=l \leq i^{*}$, and the operations of meet $\wedge$ and join $\vee$ are defined as usually. The $\sigma$ completeness condition, meaning that the join of countable families of pairwise orthogonal events exists, is required in order to have a well defined theory of quantum observables over $L$.

It is important to note in relation to the proposed functorial representation of quantum event algebras that despite the fact that each object $L$ in $\mathcal{L}$ is not Boolean, there always exists an underlying categorical diagram of Boolean subalgebras of $L$, where each one of them is generated by the following well-known compatibility condition: any arbitrary pair of elements $l, \hat{l} \in L$ are compatible if the sublattice generated by $\left\{l, l^{*}, \hat{l}, l^{*}\right\}$ is a Boolean algebra, namely, if it is a Boolean sublattice. According to the preliminary conceptual analysis in Sect. 2.1, this indicates that a Boolean event algebra is structurally adaptable to a quantum event algebra since it encodes a structurally invariant context of co-measurable observables by means of their joint compatible spectral resolution.

\footnotetext{
${ }^{2} \mathrm{~A}$ concise account of the underpinnings of category theory, including an analysis of the preceding notions, can be found in the Appendix of [33].
} 
The latter feature motivates the consideration of a Boolean modeling or shaping functor of $\mathcal{L}, \mathbf{M}: \mathcal{B} \rightarrow \mathcal{L}$, which assigns to each Boolean event algebra in $\mathcal{B}$ (constitutive of a model category) the underlying quantum event algebra from $\mathcal{L}$, and to each Boolean homomorphism the underlying quantum algebraic homomorphism. Hence, $\mathbf{M}$ acts as a forgetful functor, not taking into account the extra Boolean structure of $\mathcal{B}$. We note that the Boolean modeling functor $\mathbf{M}$ is not assumed to be necessarily an inclusion functor in the target category. The crucial idea in this context is that being Boolean is a structural property, and in particular it is a property of a ring, as it is well-known from the celebrated Stone's representation theorem for Boolean algebras; whereas there is no analogous structural characterization of orthomodular lattices.

Because of the fact that an opposite-directing functor from $\mathcal{L}$ to $\mathcal{B}$ is not feasible, since a quantum event algebra cannot be realized within any Boolean event algebra, we seek for an extension of $\mathcal{B}$ into a larger categorical environment where such a realization becomes possible. This extension should conform with the intended physical semantics of adjoining a multiplicity of Boolean spectral invariants to a quantum event algebra, objectified as probing Boolean frames of the latter. In this perspective, the global information encoded in a quantum event algebra is expected to be recovered in a structure-preserving way by an appropriate sheaf-theoretic construction gluing together categorical diagrams of locally variable Boolean frames. As shown in the sequel, a topological gluing construction of this form can only take place by extending the categorical level of $\mathcal{B}$ to the categorical level of diagrams in $\mathcal{B}$, forming, in turn, the functor category Sets $^{\mathcal{B}^{o p}}$, which actually constitutes the free completion of $\mathcal{B}$ under colimits. The existence of colimits expresses in category theoretical language the basic intuition that a complex object may be conceived as arising from the structured interconnection of partially or locally defined information carriers in a specified covering system.

It is apparent that the realization of this extension process requires initially the construction of the functor category of presheaves of sets on Boolean event algebras, denoted by Sets $^{\mathcal{B}^{o p}}$, where we remind that $\mathcal{B}^{o p}$ is the opposite category of $\mathcal{B}$. The objects of Sets $^{\mathcal{B}^{o p}}$ are all functors, $\mathbf{P}: \mathcal{B}^{o p} \rightarrow$ Sets, with morphisms all natural transformations between such functors. Each object $\mathbf{P}$ in the category of presheaves $\mathbf{S e t s}^{\mathcal{B}^{\text {op }}}$ is a contravariant set-valued functor on $\mathcal{B}$, called a presheaf of sets on $\mathcal{B}[11$, p. 195]. The functor category of presheaves on Boolean event algebras, Sets $^{\mathcal{B}^{o p}}$, provides the instantiation of a structure known as topos. A topos exemplifies a well-defined notion of a universe of variable sets. It can be conceived as a local mathematical framework corresponding to a generalized model of set theory or as a generalized topological space [3].

In order to obtain a clear understanding of the structure of the functor category Sets $^{\mathcal{B}^{\text {op }}}$, we note that a functor $\mathbf{P}$ in Sets $^{\mathcal{B}^{\text {op }}}$ can be thought of as constructing an image of $\mathcal{B}$ in Sets contravariantly or as a contravariant translation of the 'language' of $\mathcal{B}$ into that of Sets. Thus, a functor $\mathbf{P}$ is a structure-preserving morphism of these categories, that is, it preserves compositions and identities. Given another such contravariant functor $\mathbf{Q}$ of $\mathcal{B}$ into Sets we need to compare them. This can be done by assigning, for each object $B$ in $\mathcal{B}$, a transformation, $\tau_{B}: \mathbf{P}(B) \rightarrow \mathbf{Q}(B)$, which compares the two images of the object $B$. Not any morphism will do, however, as we intend the construction to be parametric in $B$ rather than ad hoc. Since $B$ is an object in $\mathcal{B}$, whereas, $\mathbf{P}(B)$ is in Sets, we cannot link them by a morphism. Rather, the goal is that the transformation should respect the morphisms of $\mathcal{B}$ or, in other words, the interpretations of $f: B \rightarrow C$ by $\mathbf{P}$ and $\mathbf{Q}$ should be compatible with the transformation under $\tau$. Then $\tau$ is a natural 
transformation in the functor category of presheaves, Sets ${ }^{\mathcal{B}^{o p}}$, on Boolean event algebras.

Alternatively, an object $\mathbf{P}$ of Sets $^{\mathcal{B}^{\text {op }}}$ may be understood as a right action of the category $\mathcal{B}$ on a set of observables, which is partitioned into a variety of Boolean spectral kinds parameterized by the Boolean event algebras $B$ in $\mathcal{B}$. Such an action $\mathbf{P}$ is equivalent to the specification of a diagram in $\mathcal{B}$, to be thought of as a $\mathcal{B}$-variable set forming a presheaf $\mathbf{P}(\mathrm{B})$ on $\mathcal{B}$. For each Boolean algebra $B$ of $\mathcal{B}, \mathbf{P}(\mathrm{B})$ is a set, and for each Boolean homomorphism $f: C \rightarrow B, \mathbf{P}(f): \mathbf{P}(B) \rightarrow \mathbf{P}(\mathrm{C})$ is a set-theoretic function, such that, if $p \in \mathbf{P}(\mathrm{B})$, the value $\mathbf{P}(f)(p)$ for an arrow $f: C \rightarrow B$ in $\mathcal{B}$ is called the restriction of $p$ along $f$ and is denoted by $\mathbf{P}(f)(p)=p \cdot f$. From a physical viewpoint, the purpose of introducing the notion of a presheaf $\mathbf{P}$ on $\mathcal{B}$, in the environment of the functor category Sets $^{\mathcal{B}^{o p}}$, is to identify an element of $\mathbf{P}(\mathrm{B})$, that is, $p \in \mathbf{P}(\mathrm{B})$, with an event observed by means of a physical procedure over a Boolean domain cover for a quantum event algebra. As further analyzed in Sect. 3.2, this identification forces the interrelation of observed events, over all Boolean probing frames of the base category $\mathcal{B}$, to fulfil the requirements of a uniform and homologous fibred structure.

In the categorical setting of the functor category, $\operatorname{Sets}^{\mathcal{B}^{o p}}$, it becomes possible to realize a quantum event algebra $L$ in $\mathcal{L}$ in terms of a distinguished presheaf of Boolean event algebras, which emerges due to the invariant spectral capacity of the latter to act locally as logical probing frames of a quantum event algebra. These Boolean frames of an $L$ in $\mathcal{L}$ are objectified as $L$-targeting morphisms in $\mathcal{L}$,

$$
\psi_{B}: \mathbf{M}(B) \rightarrow L,
$$

being interrelated by the operation of presheaf restriction. This means explicitly that for each Boolean homomorphism $f: C \rightarrow B$, if $\psi_{B}: \mathbf{M}(B) \rightarrow L$ is a Boolean frame of $L$, the corresponding Boolean frame $\psi_{C}: \mathbf{M}(C) \rightarrow L$ is given by the restriction or pullback of $\psi_{B}$ along $f$, denoted by $\psi_{B} \cdot f=\psi_{C}$. Thus, we obtain a contravariant presheaf functor,

$$
\mathbf{R}(L)(-)=\operatorname{Hom}_{\mathcal{L}}(\mathbf{M}(-), L),
$$

called the functor of Boolean frames of $L$. Since the physical interpretation of the functor $\mathbf{R}(L)(-)$ refers to the functorial realization of a quantum event algebra $L$ in $\mathcal{L}$ in terms of Boolean algebras $B$ in $\mathcal{B}$, we think of $\mathbf{R}(L)(-)$ as the variable Boolean spectral functor of $L$ through the structured multitudes of local Boolean frames adjoined to it. In this manner, a Boolean event algebra $B$, induced locally by the measurement of a quantum observable via the spectral decomposition of the corresponding self-adjoint operator, acts as a partial coordinatizing logical probing frame of a quantum event algebra $L$ in terms of Boolean coefficients.

It is now important to stress that each Boolean algebra $B$ in $\mathcal{B}$ gives naturally rise to a contravariant representable Hom-functor $\mathbf{y}^{B}:=\mathbf{y}[B]:=H_{o} m_{\mathcal{B}}(-, B)$. This functor defines a $\mathcal{B}$-variable set or, equivalently, a presheaf of sets on $\mathcal{B}$ for each $B$ in $\mathcal{B}$. Concomitantly, the functor $\mathbf{y}$ is a full and faithful functor from $\mathcal{B}$ to the contravariant functors on $\mathcal{B}$, i.e.,

$$
\mathbf{y}: \mathcal{B} \longrightarrow \text { Sets }^{\mathcal{B}^{o p}},
$$

defining the Yoneda embedding $\mathcal{B} \hookrightarrow$ Sets $^{\mathcal{B}^{o p}}[2$, pp. 187-189, 39, p. 26]. According to the Yoneda Lemma, there exists an injective (one-to-one) correspondence between elements of the set $\mathbf{P}(B)$ and natural transformations in Sets ${ }^{\mathcal{B}^{o p}}$ from $\mathbf{y}[B]$ to $\mathbf{P}$ and this correspondence is natural in both $\mathbf{P}$ and $B$, for every presheaf of sets $\mathbf{P}$ in $\mathbf{S e t s}^{\mathcal{B}^{o p}}$ and 
Boolean algebra $B$ in $\mathcal{B}$. The functor category of presheaves of sets on Boolean event algebras, Sets $^{\mathcal{B}^{o p}}$, is a complete and co-complete category. Thus, the Yoneda embedding $\mathbf{y}: \mathcal{B} \longrightarrow$ Sets $^{\mathcal{B}^{o p}}$ constitutes the intended free completion of $\mathcal{B}$ under colimits of diagrams of Boolean event algebras.

The deep meaning of this fact is that if we consider a Boolean modeling functor $\mathbf{M}$ : $\mathcal{B} \rightarrow \mathcal{L}$ there exists precisely one corresponding uniquely defined, up to isomorphism, colimit-preserving functor $\widehat{\mathbf{M}}$ : Sets $^{\mathcal{B}^{o p}} \rightarrow \mathcal{L}$, such that the following diagram commutes:

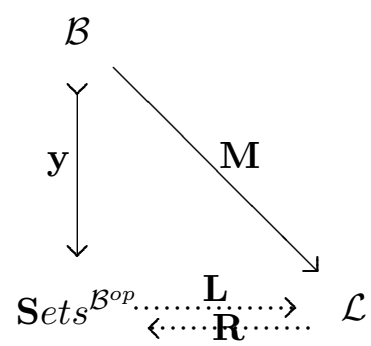

Consequently, every morphism from a Boolean algebra $B$ in $\mathcal{B}$ to a quantum event algebra $L$ in $\mathcal{L}$ factors uniquely through the functor category $\operatorname{Sets}^{\mathcal{B}^{o p}}$, and the consideration of the colimit-preserving functor $\widehat{\mathbf{M}}:$ Sets $^{\mathcal{B}^{o p}} \rightarrow \mathcal{L}$ is crucial for understanding the underlying structure of $L$ in $\mathcal{L}$ in terms of diagrams of Boolean probing frames.

The physical significance of the functor $\widehat{\mathbf{M}}$ is unraveled by the fact that it plays the role of a left adjoint $\mathbf{L}$ and, thus, colimit-preserving functor from $\mathbf{S e t s}^{\mathcal{B}^{o p}}$ to $\mathcal{L}$. More precisely, the functor $\widehat{\mathbf{M}}:=\mathbf{L}$ is the left adjoint of the categorical adjunction between the

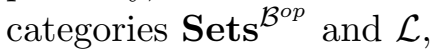

$$
\mathbf{L}: \operatorname{Sets}^{\mathcal{B}^{o p}} \rightarrow \mathcal{L}
$$

whilst the right adjoint functor,

$$
\mathbf{R}: \mathcal{L} \rightarrow \operatorname{Sets}^{\mathcal{B}^{o p}}
$$

is physically interpreted as the Boolean realization functor of $\mathcal{L}$ in terms of variable local probing frames, functioning as natural contexts for measurement of observables. The existence of the functorial relations (2.4) and (2.5) designates the fact that a quantum event algebra $L$ in $\mathcal{L}$ can be expressed in terms of structured multitudes of interlocking local Boolean frames capable of carrying all the information encoded in the former.

Henceforth, the problem of establishing a functorial representation of a quantum event algebra via Boolean frames has a universal solution, which is provided by the left adjoint functor $\mathbf{L}: \mathbf{S e t s}^{\mathcal{B}^{o p}} \rightarrow \mathcal{L}$ to the Boolean realization functor $\mathbf{R}: \mathcal{L} \rightarrow$ Sets $^{\mathcal{B}^{o p}}$. In other words, the existence of the left adjoint functor $\mathbf{L}$ paves the way for an explicit reconstruction of quantum event algebras by means of appropriate diagrams of Boolean probing frames in a structure-preserving manner, based on partial congruences between the Boolean and quantum kinds of event structure. Since our interpretative scheme of complementarity is based on this pair of adjoint functors, it is useful to express their bidirectional correspondence in the form of the following theorem, formulated and proved in [45], see also [50].

Theorem. There exists a categorical adjunction between the categories Sets $^{\mathcal{B}^{o p}}$ and $\mathcal{L}$, called the Boolean frames-quantum adjunction, established by the pair of adjoint functors 
$\mathbf{L} \dashv \mathbf{R}$ as follows:

$$
\mathbf{L}: \operatorname{Sets}^{\mathcal{B}^{o p}} \longleftarrow \mathcal{L}: \mathbf{R}
$$

where, the right adjoint, $\mathbf{R}$, is the Boolean realization functor of a quantum categorical event structure $\mathcal{L}$ in Sets $^{\mathcal{B}^{o p}}$, whereas, the left adjoint, $\mathbf{L}$, is the colimit-preserving functor providing the synthesis of a quantum categorical event structure by means of diagrams of Boolean frames.

Equivalently, there exists a bijection, which is natural in both $\mathbf{P}$ in $\operatorname{Sets}^{\mathcal{B}^{o p}}$ and $L$ in $\mathcal{L}$,

$$
\operatorname{Hom}_{\mathbf{S e t s}}{ }^{\text {oop }}(\mathbf{P}, \mathbf{R}(L)) \cong \operatorname{Hom}_{\mathcal{L}}(\mathbf{L P}, L)
$$

abbreviated as follows:

$$
\operatorname{Nat}(\mathbf{P}, \mathbf{R}(L)) \cong \operatorname{Hom}_{\mathcal{L}}(\mathbf{L P}, L) .
$$

Corollary. The left adjoint functor of the Boolean frames-quantum adjunction, $\mathbf{L}:$ Sets $^{\mathcal{B}^{\text {op }}} \rightarrow$ $\mathcal{L}$, is defined for each presheaf $\mathbf{P}$ in $\mathbf{S e t s}^{\mathcal{B}^{o p}}$ as the colimit $\mathbf{L}(\mathbf{P})$ :

$$
\mathbf{L}(\mathbf{P})=\operatorname{Colim}\left\{\int(\mathbf{P}, \mathcal{B}) \stackrel{\int_{\mathbf{P}}}{\longrightarrow} \mathcal{B} \stackrel{\mathbf{M}}{\longrightarrow} \mathcal{L}\right\}
$$

The colimit $\mathbf{L}(\mathbf{P})$ is explicitly constructed in Sect. 3.2 in relation to the physically important case of our interest, $\mathbf{P}=\mathbf{R}(L)$, namely, when the presheaf functor $\mathbf{P}$ of Boolean event algebras represents the functor of Boolean frames $\mathbf{R}(L)$ of a quantum event algebra $L$.

We recall that the Boolean modeling functor $\mathbf{M}$ is not assumed to be necessarily an inclusion functor in the target category. Of particular significance for its precise specification it is the case where, in the bijection defining the above adjunction, we make use of the contravariant representable Hom-functor $\mathbf{y}^{B}:=\mathbf{y}[B]:=H_{\mathcal{B}}(-, B)$ on the category of Boolean event algebras $\mathcal{B}$. Then, the bijection takes the following form:

$$
\operatorname{Nat}(\mathbf{y}[B], \mathbf{R}(L)) \cong \operatorname{Hom}_{\mathcal{L}}(\mathbf{L y}[B], L) .
$$

Furthermore, it is instructive to consider the split discrete fibration induced by the representable presheaf $\mathbf{y}[B]$, where $\mathcal{B}$ is taken as the base category of the fibration. In particular, this fibration is expressed by the functor

$$
\int_{\mathbf{y}[B]}: \int(\mathbf{y}[B], \mathcal{B}) \rightarrow \mathcal{B}
$$

i.e. by the projection on the second coordinate of the category of elements $\int(\mathbf{y}[B], \mathcal{B})$ of $\mathbf{y}[B]$. Since $\mathbf{y}[B]$ is a representable functor, the above category of elements of $\mathbf{y}[B]$ has a terminal object, i.e. the element $1: B \rightarrow B$ of $\mathbf{y}[B](B)$. Therefore, the colimit of the composite $\mathbf{M} \circ \int_{\mathbf{y}[B]}$ is going to be just the value of $\mathbf{M} \circ \int_{\mathbf{y}[B]}$ on the terminal object. Thus, we have:

$$
\mathbf{L y}[B] \cong \mathbf{M} \circ \int_{\mathbf{y}[B]}\left(B, 1_{B}\right)=\mathbf{M}(B) .
$$

Hence, we characterize the application of the modeling functor on a Boolean event algebra $B$, i.e. $\mathbf{M}(B)$, by means of the colimit of the representable presheaf $\mathbf{y}[B]$ on the category of Boolean event algebras. This is precisely equivalent to the assertion that the following diagram commutes: 


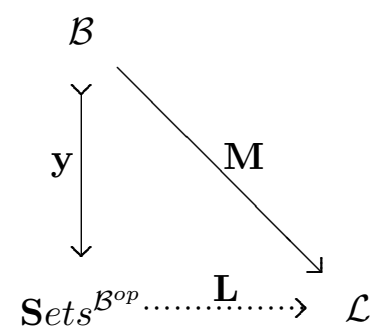

The Boolean frames-quantum adjunction, specified by the pair of adjoint functors $\mathbf{L} \dashv \mathbf{R}$, formalizes categorically the process of encoding and decoding information between diagrams of Boolean event algebras $B$ and quantum event algebras $L$ via the action of Boolean probing frames $\psi_{B}: \mathbf{M}(B) \rightarrow L$. In general, the existence of an adjunction between two categories always gives rise to a family of universal morphisms, called unit and counit of the adjunction, one for each object in the first category and one for each object in the second. In this way, each object in the first category induces a certain property in the second category and the universal morphism carries the object to the universal for that property. Most significantly, every adjunction extends to an adjoint equivalence of certain subcategories of the initial functorially correlated categories. It is precisely this categorytheoretic fact which determines the necessary and sufficient conditions for the isomorphic representation of a quantum event algebra $L$ in $\mathcal{L}$ by means of suitably qualified functors of Boolean probing frames.

We note for this purpose that every categorical adjunction is completely characterized by the unit and counit natural transformations, acquiring the status of universal mapping properties [2, pp. 208-215]. In relation to the Boolean frames-quantum adjunction, for any presheaf $\mathbf{P}$ of Boolean event algebras in the functor category $\mathbf{S e t s}^{B^{o p}}$, the unit of the adjunction is defined by

$$
\delta_{\mathbf{P}}: \mathbf{P} \longrightarrow \mathbf{R L P} .
$$

On the other side, for each quantum event algebra $L$ in $\mathcal{L}$, the counit is defined by

$$
\epsilon_{L}: \mathbf{L R}(L) \longrightarrow L
$$

As further analyzed in Sect. 3.3, the representation of a quantum event algebra $L$ in $\mathcal{L}$, in terms of the functor of Boolean frames $\mathbf{R}(L)$ of $L$, is full and faithful if and only if the counit of the Boolean frames-quantum adjunction is a quantum algebraic isomorphism, that is structure preserving, injective and surjective. In turn, the counit of the Boolean frames-quantum adjunction is a quantum algebraic isomorphism if and only if the right adjoint functor $\mathbf{R}$ is full and faithful.

We argue in the sequel that the prescribed universal solution incorporates the semantics of a twofold complementarity scheme extending the standard conception of complementarity as it has been exemplified from a contemporary viewpoint in the introductory part of this work. 


\section{Twofold Complementarity Scheme via the Boolean Frames - Quantum Adjunction}

\subsection{Towards a twofold conception of complementarity}

For methodological purposes, it is instructive initially to recall from Sect. 1 that, according to structural features of the complementarity concept, the consideration of an experimental arrangement, abstracted as the context of a Boolean frame of measurement, may serve as a referent of the assignment of an observable to a quantum system. Since the concept of a Boolean frame is qualified, by definition, as a probing frame of a quantum event algebra, it is natural to associate the semantics of complementarity with their function. In this way, every event is conditioned by at least one Boolean frame and every Boolean probing frame is constitutive of an empirical manifestation or phenomenon.

The standard formulation of complementarity from this viewpoint pertains to any two mutually incompatible Boolean frames, which correspond physically to mutually exclusive experimental arrangements, associated to canonically conjugate pairs of quantum observables. These Boolean frames neither share any common projections nor have any common refinement and, thus, may be considered as totally disjoint. Undoubtedly, the requirement of total disjointness represents an extreme case, which precludes the physically important realistic possibility of partial compatibility between various probing frames. The relaxation of such a requirement is inherently incorporated in our approach by receiving the form of a weaker version of complementarity that refers to partially compatible Boolean frames, which may have a nontrivial overlap or a common refinement or, more generally, being compatible under a pullback operation.

In order to substantiate the proposed category-theoretic analysis of complementarity, including the standard perception as a special case, we need to examine in detail the semantics of the derived universal solution concerning the functorial representation of a quantum event algebra in terms of Boolean logical probes. As a prelude to our considerations, it is significant to stress that the proposed conception of complementarity does not only pertain to the mutual relations between any two Boolean event algebras considered merely as Boolean subalgebras of a given quantum event algebra, but it pertains also to their mutual relations in their capacity to jointly act in a compatible way as Boolean probing frames of an otherwise empirically inaccessible quantum event algebra. It is the latter interpretation that is implemented by the functorial representation of a quantum event algebra in contradistinction to the former one which requires only the subalgebra relation. This differentiation is crucial because it implicates that complementarity is not only a relation between Boolean probes, or contexts of co-measurable observables, but it is primarily a reciprocal relation between two different structural kinds of event structure that can be brought into partial congruence by means of the established adjunction. This also bears as a consequence that the quantum structural kind can now be inductively constituted by the adjunction of coherently interconnected families of partially congruent Boolean probing frames covering it entirely. In this manner, the mutual relations between any two Boolean event algebras, in their capacity to function jointly as probing frames of a quantum event algebra, are constrained globally by the pair of adjoint functors $\mathbf{L} \dashv \mathbf{R}$, acting as inverse conceptual bridges between two different categorical event structures. Henceforth, these mutual relations are globally conditioned by their universal factorization through the action of the left adjoint $\mathbf{L}$ on the functor of Boolean frames $\mathbf{R}(L)$ of $L$. In 
a nutshell, one should refrain from identifying, at the risk of conflating, the notion of contextuality pertaining to the structural kind of Boolean event algebras with a global conception of complementarity pertaining to the partial functorial congruence between the Boolean and quantum kinds of event structure.

It will become clear in the sequel that the notion of contextuality, if transplanted functorially from the Boolean to the quantum kind via the modeling functor $\mathbf{M}$, gives rise to a restricted/local conceptual dimension of complementarity, which, nevertheless, is subordinate to the generalized/global dimension. As explicitly shown in Sect. 3.3, this subordination is expressed by the Boolean frames-quantum adjunction via the intervention of a covering functor of a quantum event algebra $L$, objectified as an appropriate subfunctor $\mathbf{T}$ of the functor of Boolean frames $\mathbf{R}(L)$ of $L$, i.e., $\mathbf{T} \hookrightarrow \mathbf{R}(L)$. Consequently, the proposed weakening of the former rigid notion of complementarity, implicated by the pertinent pair of adjoint functors, is qualified by means of a twofold scheme consisting of a local and a global conceptual dimension, where, importantly, the former conception is not adhoc but is functorially constrained by the latter.

\subsection{Global conceptual dimension of complementarity}

The global dimension of complementarity is formally characterized by the counit natural transformations of the identity functor in the category of quantum event algebras $\mathcal{L}$. As pointed out in the preceding section, for each quantum event algebra $L$ in $\mathcal{L}$, the counit is defined as follows:

$$
\epsilon_{L}: \mathbf{L R}(L) \longrightarrow L .
$$

Then, the counit natural transformation $\epsilon_{L}$ defines the spectral constitution of a quantum event algebra $L$ in $\mathcal{L}$ via the colimiting-interconnection of Boolean probing frames of $L$, whose domains are partially congruent Boolean event algebras to $L$.

It is clear from the counit expression (3.1) that the functorial representation of a quantum event algebra $L$ in $\mathcal{L}$ through the category Sets $^{\mathcal{B}^{o p}}$ requires an explicit calculation of the colimit $\mathbf{L R}(L)$, when the functor on which it acts is the presheaf functor of Boolean frames $\mathbf{R}(L)$ of $L$. Let us initially note that in order to calculate, in general, the colimit $\mathbf{L}(\mathbf{P})$ for any presheaf functor $\mathbf{P}$ in $\mathbf{S e t s}^{\mathcal{B}^{o p}}$, it is necessary to specify the index or parameterizing category corresponding to the functor $\mathbf{P}$, which is defined over the base category of Boolean event algebras $\mathcal{B}$. This index category is called the category of elements of the presheaf $\mathbf{P}$, denoted by $\int(\mathbf{P}, \mathcal{B})$, and defined as follows: it has objects all pairs $(B, p)$ and morphisms $(\dot{B}, \dot{p}) \rightarrow(B, p)$ are those morphisms $u: \dot{B} \rightarrow B$ of $\mathcal{B}$ for which $p \cdot u=\dot{p}$, that is, the restriction or pullback of $p$ along $u$ is $p$. Projection on the second coordinate of $\int(\mathbf{P}, \mathcal{B})$ defines a functor $\int_{\mathbf{P}}: \int(\mathbf{P}, \mathcal{B}) \rightarrow \mathcal{B}$ called the split discrete fibration induced by $\mathbf{P}$, where $\mathcal{B}$ is the base category of the fibration, as in the diagram below.

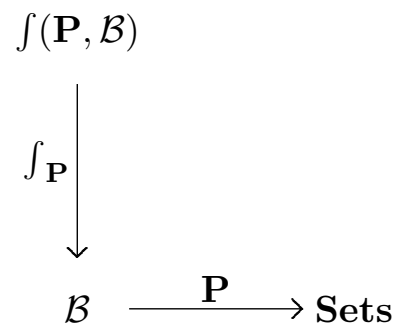


Notice that the fibers of this fibration are categories in which the only arrows are identity arrows, i.e., they are actually sets. Then, if $B$ is an object of $\mathcal{B}$, the inverse image under $\int_{\mathbf{P}}$ of $B$ is simply the set $\mathbf{P}(B)$, although its elements are written as pairs so as to form a disjoint union.

From a physical viewpoint, the split discrete fibration induced by $\mathbf{P}$ provides a welldefined notion of a uniform homologous fibred structure in the following sense. Firstly, by the arrows specification defined in the category of elements of $\mathbf{P}$, any element $p$, determined over the reference locus $B$, is homologously related with any other element $\not p$ over the reference locus $B$, and so on, by variation over all the reference loci of the base category $\mathcal{B}$. Secondly, all the elements $p$ of $\mathbf{P}$ of the same sort $B$, namely, the elements determined over the same reference locus $B$, are uniformly equivalent to each other, since all the arrows in $\int(\mathbf{P}, \mathcal{B})$ are induced by lifting arrows from the base $\mathcal{B}$.

The task of calculating the colimit $\mathbf{L R}(L)$, when the presheaf functor $\mathbf{P}$ represents the functor of Boolean frames $\mathbf{R}(L)$ of a quantum event algebra $L$, is simplified by the observation that there exists an underlying, colimit-preserving, faithful functor from the category $\mathcal{L}$ to the category Sets. Thus, we can calculate the colimit by means of set-valued equivalence classes in Sets, under the constraint that the derived set of equivalence classes carries the structure of a quantum event algebra.

In this connection, it is instructive to recall from Sect. 2.2 that the Boolean realization functor of the category of quantum event algebras $\mathcal{L}$ into the category of presheaves of Boolean event algebras Sets $^{\mathcal{B}^{o p}}$ is defined by

$$
\mathbf{R}: \mathcal{L} \rightarrow \operatorname{Sets}^{\mathcal{B}^{o p}} .
$$

Then, the functor of Boolean frames $\mathbf{R}(L)$ of a quantum event algebra $L$ in $\mathcal{L}$ is the image of the realization functor $\mathbf{R}$, evaluated at $L$, into the category of presheaves of Boolean event algebras Sets $^{\mathcal{B}^{o p}}$, i.e.,

$$
\mathbf{R}(L): \mathcal{B}^{o p} \rightarrow \text { Sets } .
$$

Thus, the functor $\mathbf{R}(L)$ of $L$ is actually an object in the category of presheaves Sets $^{\mathcal{B}^{o p}}$, representing $L$ in the environment of the topos of presheaves over the base category $\mathcal{B}$.

It is important to stress that the Boolean realization functor of a quantum categorical event structure $\mathcal{L}$ is completely determined by the action of the functor of Boolean frames, for each quantum event algebra $L$ in $\mathcal{L}$, on the objects and arrows of the category of Boolean event algebras $\mathcal{B}$. It follows immediately from Eq. (2.2) that its action on a Boolean algebra $B$ in $\mathcal{B}$ is given by

$$
\mathbf{R}(L)(B)=\operatorname{Hom}_{\mathcal{L}}(\mathbf{M}(B), L),
$$

while its action on a Boolean homomorphism $x: D \longrightarrow B$ in $\mathcal{B}$, for $v: \mathbf{M}(B) \longrightarrow L$ is defined by

$$
\begin{aligned}
& \mathbf{R}(L)(x): \operatorname{Hom}_{\mathcal{L}}(\mathbf{M}(B), L) \longrightarrow \operatorname{Hom}_{\mathcal{B}}(\mathbf{M}(D), L), \\
& \mathbf{R}(L)(x)(v)=v \circ x .
\end{aligned}
$$

As already pointed out, the functor of Boolean frames $\mathbf{R}(L)$ of a quantum event algebra $L$ in $\mathcal{L}$ forms a presheaf of sets on Boolean event algebras $B$ in $\mathcal{B}$. Thus, in complete analogy with the preceding analysis concerning any presheaf functor $\mathbf{P}$ in $\operatorname{Sets}^{\mathcal{B}^{\text {op }}}$, we 
consider the corresponding category of elements $\int(\mathbf{R}(\mathbf{L}), \mathcal{B})$, specified as follows: it has objects all pairs $\left(B, \psi_{B}\right)$, where $B$ is a Boolean event algebra and $\psi_{B}: \mathbf{M}(B) \rightarrow L$ is a Boolean probing frame of $L$ defined over $B$. The morphisms of $\int(\mathbf{R}(\mathbf{L}), \mathcal{B})$, denoted by $\left(\dot{B}, \psi_{\dot{B}}\right) \rightarrow\left(B, \psi_{B}\right)$, are those Boolean event algebra homomorphisms $u: \dot{B} \rightarrow B$ of the base category $\mathcal{B}$ for which $\psi_{B} \cdot u=\psi_{\dot{B}}$, that is, the restriction or pullback of the Boolean frame $\psi_{B}$ along $u$ is $\psi_{\dot{B}}$. Then, the projection functor $\int_{\mathbf{R}(L)}: \int(\mathbf{R}(\mathbf{L}), \mathcal{B}) \rightarrow \mathcal{B}$, namely, the split discrete fibration induced by the functor of Boolean frames of $L$, where $\mathcal{B}$ is the base category of the fibration, provides, as previously explained, a uniform and homologous fibred representation of quantum events in terms of Boolean probing frames for the measurement of observables. The notion of functional dependence incorporated in this representation forces a global quantum event algebra to be partitioned into sorts parameterized by the Boolean frames of the base category $\mathcal{B}$. In this way, the functioning of a Boolean localization scheme on a global structure of quantum events is represented by means of a fibred construct, understood geometrically as a variable set of possible events over the base category of local Boolean probing frames conditioning the actualization of events.

Consequently, the index category corresponding to the functor $\mathbf{R}(L)$ is the category of its elements $\mathcal{I} \equiv \int(\mathbf{R}(\mathbf{L}), \mathcal{B})$, whence the functor $\left[\mathbf{M} \circ \int_{\mathbf{R}(L)}\right]$ defines the diagram $\mathcal{I} \rightarrow \mathcal{L}$ over which the colimit should be calculated. Hence, we obtain:

$$
\mathbf{L R}(L)=\mathbf{L}_{\mathbf{M}}(\mathbf{R}(L))=\operatorname{Colim}\left\{\int(\mathbf{R}(\mathbf{L}), \mathcal{B}) \stackrel{\int_{\mathbf{R}(L)}}{\longrightarrow} \mathcal{B} \stackrel{\mathbf{M}}{\longrightarrow} \mathcal{L} \rightarrow \text { Sets }\right\}
$$

It has been shown in [50] that the sought colimit is equivalent to the definition of the following tensor product structure,

$$
\mathbf{L R}(L) \cong \mathbf{R}(L) \otimes_{\mathcal{B}} \mathbf{M},
$$

formed by the set-valued functors,

$$
\mathbf{R}(L): \mathcal{B}^{o p} \rightarrow \text { Sets, } \quad \mathbf{M}: \mathcal{B} \rightarrow \text { Sets },
$$

where the contravariant functor $\mathbf{R}(L)$ is considered as a right $\mathcal{B}$-module and the covariant functor $\mathbf{M}$ as a left $\mathcal{B}$-module, in complete analogy with the algebraic definition of the tensor product of a right $\mathcal{B}$-module with a left $\mathcal{B}$-module over a ring of coefficients $\mathcal{B}$ [37]. We call this construction the functorial tensor product decomposition of the colimit in the category of elements of $\mathbf{R}(L)$ induced by the Boolean modeling functor $\mathbf{M}: \mathcal{B} \rightarrow \mathcal{L}$ of $\mathcal{L}$.

Thus, for a Boolean probing frame $\psi_{B} \in \mathbf{R}(L)(B), v: \dot{B} \rightarrow B$ and $\dot{q} \in \mathbf{M}(\dot{B})$, the elements of the set $\mathbf{R}(L) \otimes_{\mathcal{B}} \mathbf{M}$ are all of the form $\chi\left(\psi_{B}, q\right)$, which can be written in a suggestive notation as,

$$
\chi\left(\psi_{B}, q\right)=\psi_{B} \otimes q, \quad \psi_{B} \in \mathbf{R}(L)(B), q \in \mathbf{M}(B),
$$

such that,

$$
\psi_{B} \cdot v \otimes \dot{q}=\psi_{B} \otimes v(\dot{q}), \quad \psi_{B} \in \mathbf{R}(L)(B), \dot{q} \in \mathbf{M}(\dot{B}), v: \dot{B} \rightarrow B .
$$

This state of affairs gives rise to the conclusion that the set $\mathbf{R}(L) \otimes_{\mathcal{B}} \mathbf{M}$ is actually the quotient of the set $\sum_{B} \mathbf{R}(L)(B) \times \mathbf{M}(B)$, for $B \in \mathcal{B}$, by the smallest equivalence relation generated by the above equations, whence the elements $\psi_{B} \otimes q$ of the quotient 
set $\mathbf{R}(L) \otimes_{\mathcal{B}} \mathbf{M}$ are the equivalence classes of this relation. Since the morphisms $\psi_{B}$ : $\mathbf{M}(B) \rightarrow L$ denote Boolean probing frames of $L$, encoded as elements in the associated index category, $\mathcal{I} \equiv \int(\mathbf{R}(\mathbf{L}), \mathcal{B})$, and $q$ denotes a projection operator $q \in \mathbf{M}(B)$, then, the quotient set $\mathbf{R}(L) \otimes_{\mathcal{B}} \mathbf{M}$ is actually a set of equivalence classes of pointed Boolean frames.

Henceforth, the function of the left adjoint of the Boolean frames-quantum adjunction, $\mathbf{L}:$ Sets $^{\mathcal{B}^{o p}} \rightarrow \mathcal{L}$, when it acts on the functor of Boolean frames $\mathbf{R}(L)$ of a quantum event algebra $L$, is defined, for each $L$ in $\mathcal{L}$, as the colimit $\mathbf{L R}(L)$ of Eq. (3.6), which, in turn, is equivalent to the quotient set $\mathbf{R}(L) \otimes_{\mathcal{B}} \mathbf{M}$. Furthermore, the latter set is naturally endowed with the structure of a quantum event algebra, thus, completing the construction of the left adjoint colimit in $\mathcal{L}$ via the category of Sets.

The embedding of a quantum event algebraic structure into the quotient set $\mathbf{R}(L) \otimes_{\mathcal{B}} \mathbf{M}$ is specified as follows. First, the orthocomplementation operator is defined by the assignment:

$$
\left(\psi_{B} \otimes q\right)^{*}:=\psi_{B} \otimes q^{*} .
$$

Second, the unit element is defined as:

$$
\mathbf{1}:=\psi_{B} \otimes 1 .
$$

Third, two equivalence classes in the quotient set $\mathbf{R}(L) \otimes_{\mathcal{B}} \mathbf{M}$ can be ordered if and only if they have a common refinement. Consequently, the partial order structure is defined by the assignment,

$$
\left(\psi_{B} \otimes q\right) \preceq\left(\psi_{C} \otimes r\right),
$$

if and only if,

$$
d_{1} \preceq d_{2},
$$

where we have made the following identifications,

$$
\begin{aligned}
& \left(\psi_{B} \otimes q\right)=\left(\psi_{D} \otimes d_{1}\right), \\
& \left(\psi_{C} \otimes r\right)=\left(\psi_{D} \otimes d_{2}\right),
\end{aligned}
$$

with $d_{1}, d_{2} \in \mathbf{M}(D)$, according to the pullback diagram,

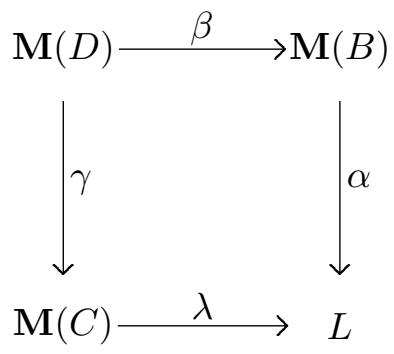

such that $\beta\left(d_{1}\right)=q, \gamma\left(d_{2}\right)=r$, and $\beta: \mathbf{M}(D) \rightarrow \mathbf{M}(B), \gamma: \mathbf{M}(D) \rightarrow \mathbf{M}(C)$ denote the pullback of $\alpha: \mathbf{M}(B) \rightarrow L$ along $\lambda: \mathbf{M}(C) \rightarrow L$ in the category of quantum event algebras. Apparently, the ordering relation between any two equivalence classes of pointed Boolean frames in the set $\mathbf{R}(L) \otimes_{\mathcal{B}} \mathbf{M}$ requires the existence of pullback compatibility conditions between the corresponding Boolean frames. This is a crucial requirement that will be also utilized in the next section for establishing the local conceptual dimension of complementarity. 
We conclude that the spectral constitution of a quantum event algebra $L$ in $\mathcal{L}$ via the Boolean frames-quantum adjunction is based on the action of the endofunctor $\mathbf{G}$ on $\mathcal{L}$, defined by

$$
\begin{gathered}
\mathbf{G}:=\mathbf{L R}: \mathcal{L} \rightarrow \mathbf{S e t s}^{\mathcal{B}^{o p}} \rightarrow \mathcal{L}, \\
L \rightarrow \mathbf{R}(L) \rightarrow \mathbf{L R}(L) \rightarrow L,
\end{gathered}
$$

which may be thus called the global spectral constitution endofunctor of a quantum categorical event structure $\mathcal{L}$ via Boolean probing frames. Note that the endofunctor $\mathbf{G}$ on $\mathcal{L}$ is obtained by the composition of the right adjoint $\mathbf{R}: \mathcal{L} \rightarrow$ Sets $^{\mathcal{B}^{o p}}$ together with the left adjoint $\mathbf{L}:$ Sets $^{\mathcal{B}^{o p}} \rightarrow \mathcal{L}$. The significance of the endofunctor $\mathbf{G}$ on $\mathcal{L}$ lies on the fact that it induces the precise natural transformation of the identity functor on $\mathcal{L}$ that gives rise to the pertinent counit universal morphism of the Boolean frames-quantum adjunction characterizing $\mathcal{L}$.

In particular, if the counit universal morphism, evaluated at $L$, for each quantum event algebra $L$ in $\mathcal{L}$, is expressed in terms of equivalence classes of pointed Boolean frames, viz. $\mathbf{L R}(L) \cong \mathbf{R}(L) \otimes_{\mathcal{B}} \mathbf{M}$, we obtain:

$$
\epsilon_{L}: \mathbf{R}(L) \otimes_{\mathcal{B}} \mathbf{M} \longrightarrow L .
$$

Thus, the counit $\epsilon_{L}$ fits into the following diagram:

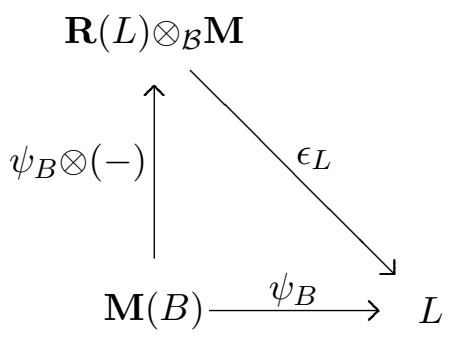

Accordingly, for every Boolean frame $\psi_{B}: \mathbf{M}(B) \rightarrow L$ the projection operator $q \in$ $\mathbf{M}(B)$ is mapped to an event in $L$ only through its factorization via the equivalence class $\psi_{B} \otimes q$ of pointed Boolean frames or, equivalently,

$$
\epsilon_{L}\left(\left[\psi_{B} \otimes q\right]\right)=\psi_{B}(q), \quad q \in \mathbf{M}(B) .
$$

This epitomizes the semantical role of the global conceptual dimension of complementarity as a relation between the Boolean and the quantum structural kinds of event structure that can be brought into partial congruence by means of the established adjunction.

\subsection{Local conceptual dimension of complementarity}

The local dimension of complementarity is formally characterized by the conditions that make the counit natural transformations of the identity functor in the category of quantum event algebras $\mathcal{L}$ an isomorphism. In turn, the counit isomorphism expresses the property of invariance of a quantum event algebra under encoding in terms of appropriate families of Boolean event algebras via probing frames in $\mathbf{R}(L)$, and then decoding back by means of the action of the left adjoint on the former, denoted by $\mathbf{L R}(L)$.

We recall that, in view of the global dimension of complementarity, the counit $\epsilon_{L}$ defines the spectral constitution of a quantum event algebra $L$ in $\mathcal{L}$ via the colimit of the 
functor of Boolean probing frames of $L$, whose domains are partially congruent Boolean event algebras to $L$. This colimit $\mathbf{L R}(L)$ is actually an object in $\mathcal{L}$, qualified as partially ordered, if additionally there exist pullback compatibility conditions between the Boolean frames that may be ordered.

We notice that if the counit evaluated at $L$ is an isomorphism, then $L$ can be considered as a fixed point of the corresponding global spectral constitution endofunctor of $L$ via Boolean probing frames. In general, the counit natural transformation $\epsilon_{L}$ is a natural isomorphism if and only if the right adjoint functor of the Boolean frames-quantum adjunction is full and faithful or, equivalently, if and only if the cocone from the functor $\mathbf{M} \circ \int_{\mathbf{R}(L)}$ to $L$ is universal for each $L$ in $\mathcal{L}$. In the latter case, we characterize the functor $\mathbf{M}: \mathcal{B} \rightarrow \mathcal{L}$ as a dense Boolean modeling functor. On this account, therefore, it is important to specify the necessary and sufficient conditions which make the counit $\epsilon_{L}$ to be an isomorphism. This requirement leads to the notion of sheaf-theoretic localization of $L$ through the probing frames $\psi_{B}: \mathbf{M}(B) \rightarrow L$, giving subsequently rise to the local conceptual dimension of complementarity.

As a first step, we emphasize that if the counit natural transformation $\epsilon_{L}$ at $L$ can be suitably restricted to an isomorphism $\mathbf{R}(L) \otimes_{\mathcal{B}} \mathbf{M} \cong L$, then the quantum structural information of $L$ can be completely encoded and classified logically through equivalence classes of pointed Boolean frames. For this purpose, we need to impose appropriate conditions on families of Boolean frames in $\mathbf{R}(L)$, which are going to function as local Boolean covers of $L$. The requirements qualifying such restricted families of Boolean frames as local Boolean covers of $L$ are the following: First, they should constitute a minimal generating class of Boolean frames instantiating a subfunctor $\mathbf{T}$ of the functor of Boolean frames $\mathbf{R}(L)$ of $L$. Second, they should jointly form an epimorphic family covering $L$ entirely on their overlaps. Third, they should be compatible under refinement operations or, more generally, pullback conditions in $L$. Fourth, they should be transitive such that subcovers of covers of $L$ can be qualified as covers themselves. We formulate these notions as follows.

A functor of Boolean coverings for a quantum event algebra $L$ in $\mathcal{L}$ is defined as a subfunctor $\mathbf{T}$ of the functor of Boolean frames $\mathbf{R}(L)$ of $L$, i.e., $\mathbf{T} \hookrightarrow \mathbf{R}(L)$. For each Boolean algebra $B$ in $\mathcal{B}$, a subfunctor $\mathbf{T} \hookrightarrow \mathbf{R}(L)$ is equivalent to an algebraic right ideal or spectral sieve of quantum homomorphisms $\mathbf{T} \triangleright \mathbf{R}(L)$, defined by the requirement that, for each $B$ in $\mathcal{B}$, the set of elements of $\mathbf{T}(B) \subseteq[\mathbf{R}(L)](B)$ is a set of Boolean frames $\psi_{B}: \mathbf{M}(B) \rightarrow L$ of $\mathbf{R}(L)(B)$, called Boolean covers of $L$, satisfying the following property:

If $\left[\psi_{B}: \mathbf{M}(B) \rightarrow L\right] \in \mathbf{T}(B)$, viz. it is a Boolean cover of $L$, and $\mathbf{M}(v): \mathbf{M}(B) \rightarrow$ $\mathbf{M}(B)$ in $\mathcal{L}$ for $v: \dot{B} \rightarrow B$ in $\mathcal{B}$, then $\left[\psi_{B} \circ \mathbf{M}(v): \mathbf{M}(\dot{B}) \rightarrow \mathcal{L}\right] \in \mathbf{T}(B)$, viz. it is also a Boolean cover of $L\}$.

A family of Boolean covers $\psi_{B}: \mathbf{M}(B) \longrightarrow L, B$ in $\mathcal{B}$, is the generator of a spectral sieve of Boolean coverings $\mathbf{T}$, if and only if, this sieve is the smallest among all containing that family. The spectral sieves of Boolean coverings for an $L$ in $\mathcal{L}$ constitute a partially ordered set under inclusion of subobjects. The minimal sieve is the empty one, namely, $\mathbf{T}(B)=\emptyset$ for all $B$ in $\mathcal{B}$, whereas the maximal sieve is the set of all probing Boolean frames of $L$ for all $B$ in $\mathcal{B}$, considered as Boolean covers.

We recall from Sect. 3.2 that the ordering relation between any two equivalence classes of pointed Boolean frames in the set $\mathbf{R}(L) \otimes_{\mathcal{B}} \mathbf{M}$ requires the existence of pullback compatibility conditions between the corresponding Boolean frames. Henceforth, if we consider a functor of Boolean coverings $\mathbf{T}$ for a quantum event algebra $L$, we require that the 
generating family of Boolean covers they belong to is compatible under pullbacks.

Then, the pullback or categorical overlap of any pair of Boolean covers $\psi_{B}: \mathbf{M}(B) \longrightarrow L$, $B$ in $\mathcal{B}$, and $\psi_{\dot{B}}: \mathbf{M}(\dot{B}) \longrightarrow L, \dot{B}$ in $\mathcal{B}$, with common codomain a quantum event algebra $L$, consists of the Boolean cover $\mathbf{M}(B) \times{ }_{L} \mathbf{M}(\dot{B})$, together with the two arrows $\psi_{B \dot{B}}$ and $\psi_{\dot{B} B}$, called projections, as shown in the diagram:

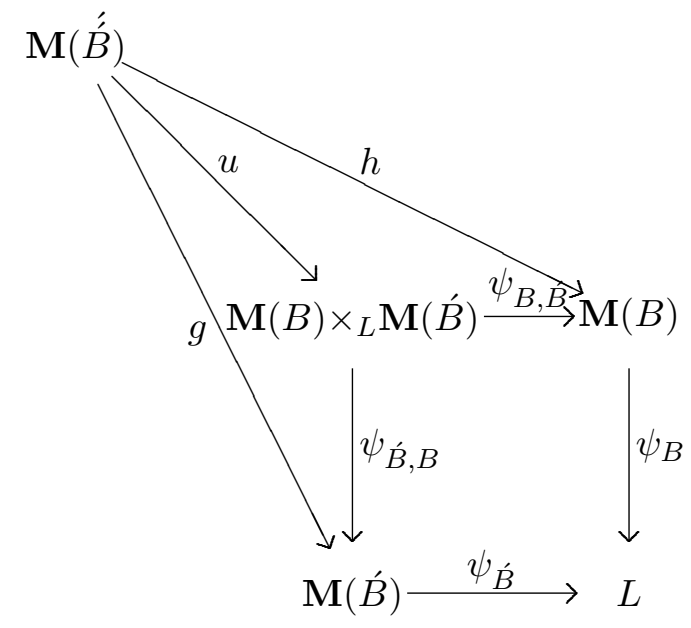

The square commutes and for any Boolean domain object $\mathbf{M}\left(\dot{B}^{\prime}\right)$ or event algebra $\dot{B}^{\prime}$ in $\mathcal{B}$ and arrows $h$ and $g$ that make the outer square commute, there is a unique $u: \mathbf{M}(\dot{B}) \longrightarrow \mathbf{M}(B) \times{ }_{L} \mathbf{M}(\dot{B})$ that makes the whole diagram commutative. Hence, we obtain the condition: $\psi_{\dot{B}} \circ g=\psi_{B} \circ h$.

We emphasize that if $\psi_{B}$ and $\psi_{\dot{B}}$ are injective morphisms, then their pullback is isomorphic with the intersection $\mathbf{M}(B) \times{ }_{L} \mathbf{M}(\dot{B})$. Then, we can define the gluing or pasting map between Boolean probing frames on their overlap, which is an isomorphism, as follows:

$$
\begin{gathered}
\Omega_{B, \dot{B}}: \psi_{\dot{B} B}\left(\mathbf{M}(B) \times{ }_{L} \mathbf{M}(\dot{B})\right) \longrightarrow \psi_{B \dot{B}}\left(\mathbf{M}(B) \times{ }_{L} \mathbf{M}(\dot{B})\right), \\
\Omega_{B, \dot{B}}=\psi_{B \dot{B}} \circ \psi_{\dot{B} B}{ }^{-1} .
\end{gathered}
$$

An immediate consequence of the preceding definition is the satisfaction of the following cocycle conditions: (i) $\Omega_{B, B}=1_{B}, 1_{B}:=i d_{B}$, (ii) $\Omega_{B, B} \circ \Omega_{\dot{B}, \dot{B}}=\Omega_{B, \dot{B}}$ if $\mathbf{M}(B) \cap \mathbf{M}(\dot{B}) \cap$ $\mathbf{M}(\dot{B}) \neq 0$, and (iii) $\Omega_{B, \dot{B}}=\Omega^{-1}{ }_{B, B}$ if $\mathbf{M}(B) \cap \mathbf{M}(\dot{B}) \neq 0$.

Thus, the gluing isomorphism $\Omega_{B, B}$ between any two Boolean frames of a spectral sieve $\mathbf{T}(L)$ assures that $\psi_{\dot{B} B}\left(\mathbf{M}(B) \times{ }_{L} \mathbf{M}(\dot{B})\right)$ and $\psi_{B \dot{B}}\left(\mathbf{M}(B) \times{ }_{L} \mathbf{M}(\dot{B})\right)$ probe $L$ on their common refinement in a compatible way. This provides the sought criterion of qualifying the local conceptual dimension of complementarity with respect to a spectral sieve $\mathbf{T}(L)$, under the proviso that the family of all Boolean covers $\psi_{B}: \mathbf{M}(B) \longrightarrow L$, for variable $B$ in $\mathcal{B}$, generating this spectral sieve, jointly form an epimorphic family covering $L$ completely,

$$
T_{L}: \sum_{\left(B_{j}, \psi_{j}: \mathbf{M}\left(B_{j}\right) \rightarrow L\right)} \mathbf{M}\left(B_{j}\right) \rightarrow L,
$$

where $T_{L}$ is an epimorphism in $\mathcal{L}$ with codomain a quantum event algebra $L$.

A sieve adjoined to a quantum event algebra $L$ constitutes a Boolean localizing spectral sieve of $L$ or, equivalently, a functor of Boolean localizations of $L$, if and only if it is 
closed with respect to an epimorphic family of Boolean covers of $L$ and the above cocycle conditions are satisfied. The conceptual significance of a Boolean localizing spectral sieve of $L$ lies on the fact that the functor of Boolean probing frames $\mathbf{R}(L)$ becomes a structure sheaf of local Boolean coefficients when restricted to such a localizing sieve. Then, it can be shown that for a dense epimorphic family of Boolean covers in a Boolean localization functor $\mathbf{T}$ of $L$, the counit of the Boolean frames-quantum adjunction is restricted to a quantum algebraic isomorphism, i.e. the counit becomes structure-preserving, injective and surjective [45]. As a consequence, the right adjoint functor of the adjunction restricted to a Boolean localization functor becomes a full and faithful functor. This proposition can be expressed more precisely in topos theoretic terminology using the technical means of the subcanonical Grothendieck topology, consisting of epimorphic covering families on the base category of Boolean event algebras. For details the interested reader should consult [47]. Consequently, $\mathcal{L}$ becomes a reflection of the category of sheaves of variable local Boolean frames Sets ${ }^{B^{o p}}$, constituting a topos, and the structure of a quantum event algebra $L$ in $\mathcal{L}$ is encoded and preserved by the action of a family of Boolean frames if and only if this family forms a Boolean localization functor of $L$.

\section{Conceptual Aspects of the Twofold Complementarity Scheme}

The existence of a categorical adjunction between the category of quantum event algebras and the category of (pre)sheaves of variable local Boolean frames incorporates the semantics of a twofold complementarity scheme, extending the original notion of complementarity, by giving rise to two interdependent conceptual routes of global and local dimension. The global dimension of complementarity acquires a meaning by relating two hierarchically different structural kinds, namely, the Boolean and the quantum kinds of event structure that can be brought into partial congruence through the established adjunctive correspondence, consisting of the pair of adjoint functors $\mathbf{L} \dashv \mathbf{R}$,

$$
\mathbf{L}: \operatorname{Sets}^{\mathcal{B}^{o p}} \longleftarrow \mathcal{L}: \mathbf{R},
$$

and the natural bijection,

$$
\operatorname{Nat}(\mathbf{P}, \mathbf{R}(L)) \cong \operatorname{Hom}_{\mathcal{L}}(\mathbf{L P}, L) .
$$

The essential functioning of the Boolean frames-quantum adjunction in extending the complementarity concept is made transparent if we consider that it provides an amphidromous mechanism of encoding and decoding information between the Boolean and quantum kind of structures respecting their distinctive form. Thus, if we think of Sets B $^{\text {op }}$ as the categorical universe of variable local Boolean frames modeled in Sets, and of $\mathcal{L}$ as the categorical universe of quantum event structures, then the left adjoint functor $\mathbf{L}:$ Sets $^{\mathcal{B}^{\circ p}} \rightarrow \mathcal{L}$ signifies an encoding process of information from the level of local Boolean algebras to the level of global quantum event algebras, whereas the Boolean realization functor $\mathbf{R}: \mathcal{L} \rightarrow$ Sets $^{\mathcal{B}^{\text {OP }}}$ signifies a decoding process in the inverse direction. In general, the content of the information cannot remain completely invariant with respect to the bi-directional mechanism of encoding-decoding information from one categorical universe to another, and conversely. Note, in this respect, that the functors $\mathbf{L}$ and $\mathbf{R}$ 
are not inverses, since neither $\mathbf{L R}$ nor $\mathbf{R L}$ need be isomorphic to an identity functor. However, there remain two alternatives for a variable set $\mathbf{P}$ over local Boolean frames, standing for a presheaf functor $\mathbf{P}$ in $\mathbf{S e t s}^{\mathcal{B}^{\text {op }}}$, to exchange information with a quantum algebra $L$. Either the content of information is transferred in quantum terms with the colimit in the category of elements of $\mathbf{P}$ translating, represented as the quantum morphism $\mathbf{L P} \rightarrow L$, or the content of information is transferred in Boolean terms with the functor of Boolean frames of $L$ translating, represented correspondingly as the natural transformation $\mathbf{P} \rightarrow \mathbf{R}(L)$. In the first case, from the perspective of $L$, information is being received in quantum terms, while in the second, from the perspective of $\mathbf{P}$, information is being sent in Boolean terms. Then, the natural bijection of relation (4.2) corresponds to the assertion that these two distinct ways of information transfer are equivalent. Thus, the fact that these two functors are adjoint underlines an amphidromous dependent variation, safeguarding that the global information encoded in a quantum kind of event structure is retrievable in a structure-preserving manner by all possible partial structural congruences with the Boolean kind of event structure that, in addition, is empirically directly accessible.

The global dimension of complementarity, therefore, concerns primarily the fact that an object behaving in terms of the quantum kind of structure is possible to be communicated in its entirety through a multilevel structure of coherently interconnected families of Boolean probing frames that, by virtue of the established adjunctive correspondence, have the potential of unfolding its meaning and, simultaneously, preserving it consistently, thus providing a complementarity-based conception of the process of quantum becoming.

The local dimension of complementarity acquires a meaning by relating at the same level multitudes of partially ordered Boolean probing frames, used as localization contexts for the measurement of observables pertaining to a quantum system and, subsequently, integrating them in Boolean localization functors. The latter may be understood as the semantic carriers of networks of internal relations among Boolean frames with respect to which the targeted quantum event structure is consistently and completely covered. The Boolean frames, interconnected in localization functors, act like complementary pattern recognition mechanisms such that complementary aspects of the same quantum system are being formed in relation to the abstractions associated with the preparation of an experimental context for extracting information about the system under investigation. In quantum mechanics the relation between the global theoretical structure and its various empirical substructures is indeed such that, depending on the type of experimental context a quantum system is brought to interact, different manifested aspects of the system are disclosed, impossible to be combined into a single picture as in classical physics, although only one type of system is concerned. Thus, by virtue of the proposed categorical approach to quantum mechanics, a quantum event structure can only be unfolded through structured multitudes of interconnected Boolean probing frames and the twofold complementarity scheme safeguards the correct communicability both at the local dimension, relating different Boolean frames at the same level, and the global dimension, linking the Boolean and the quantum hierarchical levels by means of the established categorical adjunction. Furthermore, as shown in Sect. 3.3, if the counit of the Boolean framesquantum adjunction is restricted to a Boolean localization functor, then, it is reduced to an isomorphism and, subsequently, acquires the status of a closure condition expressing the preservation of the total information content embodied in a quantum event structure, through the bi-directional process of unfolding in terms of coherently interconnected fam- 
ilies of Boolean probing frames and then enfolding back. In this way, the global structural information of a quantum event algebra can be captured homomorphically or, by restriction to a Boolean localization functor, can be completely constituted (up to isomorphism) by means of gluing together the information collected in all compatible Boolean frames in the form of appropriate equivalence classes (by the colimit construction).

In view of the preceding considerations, it is instructive to emphasize that in our categorical approach to quantum mechanics a global quantum event structure is not conceptualized as an a priori existing set-theoretic structure, already completed in itself, but it is constituted in a continuous process of extension from the local to the global level by actualization of new potential facts with respect to local Boolean frames. For, each quantum event actualized relative to a particular probing frame serves as a datum for subsequent potential actualizations, thus instantiating simultaneously a bundle of potential relations referring to this frame. Importantly, all these potential relations, namely, all relations among observables at the local level are captured by the internal relations among their underlined Boolean frames and are extended to the global quantum level through the sheaf-theoretic gluing conditions of Boolean localization functors.

The key conceptual meaning of this approach implies, therefore, the view that the quantum world can be consistently covered in an epimorphic way, and understood through a multilayered localizing structure of overlapping and interlocking Boolean frames in category-theoretic terms, to form a coherent picture of the whole in a nontrivial manner. By virtue of this scheme of interpretation, the transcendent realist assumption that knowledge of an object is achieved by forming a representation of that object as an immutable substance possessing intrinsic properties is rejected [31]. It is replaced by the realistic possibility of formulating local or partial contextual theoretical structures permitting different or overlapping physical descriptions, which are nevertheless grounded on the same actually existing object, whose sameness is characterized by the constraint that these contextual structures collectively and globally obey the closure condition of the counit isomorphism. Consequently, the proposed category-theoretic analysis of complementarity reveals that the core of the quantum structure of reality is to be sought not in its internal constitution as a set-theoretical entity endowed with inherent qualities, but rather in the form of its relationship with the Boolean kind of structure through the established network of adjoint functors between the category of presheaves of variable overlapping Boolean frames and the category of quantum event algebras.

\section{A APPENDIX}

\section{A.1 Related Work}

The major source of inspiration in what is presently called categorical or topos-theoretic approaches to the foundations of quantum physics emanates from the 1998-1999 work of Isham and Butterfield (IB) $[28,29]$, who provided a concrete topos reformulation of the Kochen-Specker theorem, albeit proposals targeting the utility and relevance of these new mathematical methods appeared earlier (e.g., [42]). For this purpose, they considered the partially ordered set (poset) of commutative von Neumann subalgebras of the noncommutative algebra of all bounded operators on a quantum Hilbert space as a "category 
of contexts" where the only arrows are inclusions. The latter "category of contexts" served as a base category for defining the topos of presheaves of sets over the poset of commutative subalgebras. This topos-theoretic initiative has been extended, elaborated and developed further around ten years later by Döring and Isham (DI) (e.g., [20] and references therein). The central principle of their work is that the construction of a theory of physics, like quantum mechanics, is equivalent to finding a representation in "a topos of a certain formal language" that is attached to the system. Conceptually, the (DI) scheme is based on Lawvere's notion of an "elementary topos", i.e., the logical embodiment of the topos concept, serving as a generalized model of set theory being equipped with a subobject classifier, specified by a distributive Heyting algebra forcing an intuitionistic type of semantics [30].

Shortly afterwards, in relation to the (IB) approach, appeared an alternative topostheoretic approach to the foundations of quantum physics by the first author [44], which has been motivated, not by the notion of an "elementary topos", as in the (IB) case, but by the notion of a "Grothendieck topos" [39]. Since 2000, this approach has been developed in a series of works targeting the observable [45, 46], measure-theoretic [47], topological [48], geometric [49], logical [50], semantical [33] and conceptual-philosophical [21, 33, 34, $50]$ aspects of quantum theory. The notion of a "Grothendieck topos" interprets a topos as a generalized geometric environment, which makes it possible to constitute the structural information content of certain global objects, like quantum event algebras, from the nontrivial and non-classical localization properties of observables, which are utilized to "cover" locally these global objects in terms of interconnected multitudes of measurement contexts at various overlapping scales. This form of synthetic amalgamation of a global quantum object from the joint, pairwise compatible, and colimiting gluing of locally congruent with it Boolean objects culminates in the concept of a sheaf defined over a base category of Boolean frames. It should be emphasized that in contradistinction to the (IB) and (DI) approach who consider only the partial ordering of "contexts" as their base category, the full category of complete Boolean algebras with Boolean homomorphisms is used as a category of possible frames (by virtue of the spectral theorem) without any restriction to inclusions or injective morphisms. Most important, in our categorical approach to quantum mechanics, the "Grothendieck topos" interpretation is founded on the existence of a categorical adjunction between the category of presheaves of variable Boolean probing frames and the category of quantum event algebras. As systematically developed in the present paper, it is precisely the semantics of the resulting pair of adjoint functors $(\mathbf{L} \dashv \mathbf{R})$ that gives rise to the proposed re-conceptualization of the complementarity principle in quantum meshanics. The basic underlying idea formalized by this adjunctive pair of functors concerns the partial or local structural congruence between the Boolean and quantum levels of event structure as well as the encoding and decoding bridges from one to the other. The congruence relations are induced by the joint action of Boolean covering families of local frames on a global quantum event algebra at various layers of "observable resolution". The left and right adjoint functors in this Boolean framesquantum adjunction subsume the role of natural bridges between the Boolean and quantum structural levels shedding new light on the complementarity principle via a local and a global conceptual dimension interpreted sheaf-theoretically.

In this connection, there seems to exist an apparent affinity with another topostheoretic approach, called "Bohrification", by Heunen, Landsman and Spitters (HLS) circa 2010 ([24] and references therein). This approach has been an outgrowth of the (DI) 
program motivated by the adoption of a dual covariant perspective. They construct an internal commutative $C^{*}$-algebra (or an internal Boolean algebra) within the topos of copresheaves over the poset of "classical contexts", so their topos comes equipped with an "internal commutative algebraic object", which is interpreted, to some extent, as a formal expression of Bohr's doctrine of classical concepts. In relation to both (DI) and (HLS) frameworks, it has to be observed that the partial order relation of "classical contexts" is not adequate to capture the global structural information of a quantum event structure, and at least, the inclusion morphisms of the "Boolean contexts" to the quantum lattice should be also taken into account. In comparison, the present topos-approach, not based on the partial order relation of "Boolean contexts", specifies globally a quantum event structure via the left-adjoint functor of the established adjunction.

Our particular interest to the "Bohrification program" here stems from the fact that it has been applied recently by Heunen [25] as a means to explicate the complementarity principle in quantum mechanics. So it is quite elucidating to point out a fundamental difference underlying our respective perspectives and interpretation of this principle. According to Heunen, "complete complementarity" refers to taking all pairwise partially compatible classical contexts together. Specifically, given a certain $C^{*}$-algebra $A$, representing the observables of a physical system, Heunen's notion of "complete complementarity" is formalized by collecting all commutative $C^{*}$-subalgebras $C$ of $A$ into a single mathematical object, a partially ordered set $\mathcal{C}(A)$, whose partial ordering is given by set-theoretic inclusion. Each $C$ in $\mathcal{C}(A)$ represents a classical or experimental context, which has been disconnected from the others, except for the inclusion relations which relate compatible experiments. Then, "complete complementarity" means that it suffices to examine all commutative subalgebras (or "classical contexts") $C$ to determine the empirical content of the system modeled by the initial algebra $A$. It is instructive to note, however, that this construction applies in an identical manner even when $A$ itself is commutative. Otherwise, should $A$ be non-commutative, then the best one can do is to approximate it with ever larger commutative subalgebras $C$. Hence, it is clear that $\mathcal{C}(A)$ in itself is not adequate to reconstruct $A$. In a purely mathematical setting, $\mathcal{C}(A)$ may be seen as a useful tool for classifying $C^{*}$-algebras through the operation of set-theoretic inclusion, but it cannot be appropriately regarded as the mathematical space of complementarity, the latter requiring the existence of pairs of incompatible observables standing in a complementary relation in each other's determination. In our view, the preceding notion of "complete complementarity" can be rather considered as an elaboration of contextuality in relation to a quantum structure; it does not qualify properly the meaning of quantum complementarity. No doubt, complementarity embraces the notion of contextuality, but the principle of complementarity is neither reduced to contextuality nor exhausted by it. The reason is that partial compatibility among "classical contexts" within an overall quantum structure can be properly described in local sheaf-theoretic terms. In the absence of the crucial local-global distinction, the complementarity principle can be wrongly conflated with the notion of contextuality, since there is no functorial bridge of transferring information from the level of local contexts to the hierarchically different level of quantum structures and inversely. For this reason, we distinguish between a local and a global conceptual dimension of the complementarity principle, which only if considered together, they convey the essential meaning of this principle, formalized by means of a pair of adjoint functors.

It is worth mentioning, in passage, that there have been also developed recently other sheaf-theoretic and categorical approaches for studying, for instance, the issue of non- 
locality in relation to contextuality (e.g., [1]), providing context-dependent operations in logic (e.g., [19]), and representing various physical processes (e.g., [16]). Finally, we note that the interested reader may find particularly helpful the Appendix A.1. in [50], where it is presented a detailed comparison of the technical part of our category-theoretic approach with all other major categorical approaches to the foundations of quantum physics, including those mentioned above.

\section{References}

1. Abramsky, S., Brandenburger, A.: The sheaf-theoretic structure of non-locality and contextuality. New J. Phys. 13, 113036 (2011)

2. Awodey, S.: Category Theory (2nd ed.). Oxford University Press, Oxford (2010)

3. Bell, J.L.: Toposes and Local Set Theories. Dover, New York (1988/2008)

4. Beller, M.: Quantum Dialogue: The Making of a Revolution. University of Chicago Press, Chicago (1999)

5. Bohr, N.: The quantum postulate and the recent development of atomic theory. Nature (suppl.) 121, 580-590 (1928). Reprinted in J. Kalckar (ed.) Niels Bohr Collected Works, Vol. 6: Foundations of Quantum Physics I (1926-1932), pp. 109-136. North-Holland, Amsterdam (1985)

6. Bohr, N.: Can quantum-mechanical description of physical reality be considered complete? Phys. Rev. 38, 696-702 (1935)

7. Bohr, N.: Causality and complementarity. Phil. Sci. 4, 289-298 (1937)

8. Bohr, N.: The causality problem in atomic physics. In New Theories in Physics, pp. 11-30. International Institute of Intellectual Co-operation, Paris (1939). Reprinted in J. Kalckar (1996) (ed.) Niels Bohr Collected Works, Vol. 7: Foundations of Quantum Physics II (1933-1958), pp. 303-322 . North-Holland, Amsterdam (1996)

9. Bohr, N.: On the notion of causality and complementarity. Dialectica 2, 312-319 (1948)

10. Bohr, N.: Light and life revisited. In Niels Bohr, Essays 1958-1962 on Atomic Physics and Human Knowledge, pp. 23-29. Interscience, London (1963)

11. Borceux, F.: Handbook of Categorical Algebra 3: Categories of Sheaves. Encyclopedia of Mathematics and its Applications 52. Cambridge University Press, Cambridge (1994/2008)

12. Bramon, A., Garbarino, G., Hiesmayr, B.C.: Quantitative complementarity in two path interferometry. Phys. Rev. A 69, 022112 (2004)

13. Busch, P., Grabowski, M., Lahti, P.: Operational Quantum Physics. Springer, Berlin (1995)

14. Busch, P., Shilladay, C.: Complementarity and uncertainty in Mach-Zehnder interferometry and beyond. Phys. Rep. 435, 1-31 (2006)

15. Chiara, M.D., Giuntini, R., Greechie, R.: Reasoning in Quantum Theory: Sharp and Unsharp Quantum Logics. Kluwer, Dordrecht (2004)

16. Coecke, B.: Quantum picturalism. Contemp. Phys. 51(1), 59-83 (2010)

17. Coles, P.J.: Role of complementarity in superdense coding. Phys. Rev. A 88, 062317 (2013) 
18. Dieks, D.: Niels Bohr and the formalism of quantum mechanics. In: Folse, H., Faye, J. (eds.) Niels Bohr and Philosophy of Physics: Twenty-First Century Perspectives, pp. 303334. Bloomsbury Academic, London (2017)

19. Domenech, G., Freytes, H.: Contextual logic for quantum systems. J. Math. Phys. 46, $012102(2005)$

20. Döring, A., Isham, C.J.: "What is a thing?": Topos theory in the foundations of physics. In: Lecture Notes in Physics 813, pp. 753-937. Springer, Berlin (2011)

21. Epperson, M., Zafiris, E.: Foundations of Relational Realism: A Topological Approach to Quantum Mechanics and the Philosophy of Nature. Book series: Contemporary Whitehead Studies. Lexington Books, New York (2013)

22. Faye, J., Folse, H. (eds.): Niels Bohr and contemporary philosophy. Kluwer, Dordrecht (1994)

23. Gisin, N., Ribordy, G., Tittel, W., Zbinden, H.: Quantum cryptography. Rev. Mod. Phys. 74, $145(2002)$

24. Heunen, C., Landsman, N.P., Spitters, B.: Bohrification. In: Halvorson, H. (ed.) Deep Beauty, pp. 271-313. Cambridge University Press, New York (2011)

25. Heunen, C.: Complementarity in categorical quantum mechanics. Found. Phys. 42, 856-873 (2012)

26. Hilgevoord, J., Uffink, J.: The uncertainty principle. In: Zalta, E. (ed.) The Stanford Encyclopedia of Philosophy (2016)

https://plato.stanford.edu/archives/win2016/entries/qt-uncertainty/

27. Howard, D.: Who invented the 'Copenhagen interpretation'? A study in mythology. Phil. Sc. 71, 669-682 (2004)

28. Isham, C.J., Butterfield, J.: A topos perspective on the Kochen-Specker theorem: I. Quantum states as generalised valuations. Int. J. Theor. Phys. 37, 2669-2733 (1998)

29. Isham, C.J., Butterfield, J.: A topos perspective on the Kochen-Specker theorem: II. Conceptual aspects, and classical analogues. Int. J. Theor. Phys. 38, 827-859 (1999)

30. Johnstone, P.T.: Sketches of an Elephant: A Topos Theory Compendium. Vols. 1-2. Clarendon Press, London (2002)

31. Karakostas, V.: Realism and objectivism in quantum mechanics. J. Gen. Phil. Sci. 43, 45-65 (2012)

32. Karakostas, V.: Correspondence truth and quantum mechanics. Axiomathes 24, 343-358 (2014)

33. Karakostas, V., Zafiris, E.: Contextual semantics in quantum mechanics from a categorical point of view. Synthese 194, 847-886 (2017)

34. Karakostas, V., Zafiris, E.: On the structure and function of scientific perspectivism in quantum mechanics. arXiv:1806.08788 [quant-ph] (2018)

35. Kochen, S., Specker, E.P.: The problem of hidden variables in quantum mechanics. J. Math. Mech. 17, 59-87 (1967)

36. Lahti, P.J.: Uncertainty and complementarity in axiomatic quantum mechanics. Int. J. Theor. Phys. 19, 789-842 (1980)

37. Lam, T.X.: Lectures on Modules and Rings. Springer, New York (1999)

38. Ma, X., Kofler, J., Zeilinger, A.: Delayed-choice gedanken experiments and their realizations. Rev. Mod. Phys. 88, 015005 (2016) 
39. MacLane, S., Moerdijk, I.: Sheaves in Geometry and Logic: A First Introduction to Topos Theory. Springer, New York (1992)

40. Murdoch, D.: Niels Bohr's Philosophy of Physics. Cambridge University Press, Cambridge (1987)

41. Scheibe, E.: The Logical Analysis of Quantum Mechanics. Pergamon Press, Oxford (1973)

42. Selesnick, S.: Correspondence principle for the quantum net. Int. J. Theor. Phys. 30(10), 1273-1292 (1991)

43. Svozil, K.: Physical (A)Causality. Fundamental Theories of Physics 192. Springer Open (2018)

44. Zafiris, E.: Probing quantum structure with Boolean localization systems. Int. J. Theor. Phys. 39(12), 2761-2778 (2000)

45. Zafiris, E.: Boolean coverings of quantum observable structure: A setting for an abstract differential geometric mechanism. J. Geom. Phys. 50, 99-114 (2004)

46. Zafiris, E.: Interpreting observables in a quantum world from the categorial standpoint. Int. J. Theor. Phys. 43(1), 265-298 (2004)

47. Zafiris, E.: Sheaf-theoretic representation of quantum measure algebras. J. Math. Phys. 47, $092103(2006)$

48. Zafiris, E.: Generalized topological covering systems on quantum events structures. J. Phys. A, Math. Gen. 39, 1485-1505 (2006)

49. Zafiris, E: Quantum observables algebras and abstract differential geometry: The topostheoretic dynamics of diagrams of commutative algebraic localizations. Int. J. Theor. Phys. 46(2), 319-382 (2007)

50. Zafiris, E., Karakostas, V.: A categorial semantic representation of quantum event structures. Found. Phys. 43, 1090-1123 (2013)

51. Zhu, H.: Information complementarity: A new paradigm for decoding quantum incompatibility. Scient. Rep. 5, 14317 (2015) 